\title{
NGHIÊN CỨU MỘT SỐ GIẢI PHÁP NÂNG CAO HIỆU QUẢ CÔNG TÁC TRẮC ĐỊA THI CÔNG NHÀ SIÊU CAO TẦG Ở VIÊTT NAM
}

\author{
DIÊM CÔNG TRANG ${ }^{(1)}$, PHAM THANH THẠO(2), LẠI NAM THÁl(1) \\ ${ }^{(1)}$ Viện Khoa học Công nghệ Xây dựng, ${ }^{(2)}$ Trường Đại học Mỏ - Địa chất
}

\section{Tóm tắt:}

Trong những năm gần đây các dự án xây dựng các công trình nhà siêu cao tầng được triển khai rộng rãi tại Hà Nội, Tp. Hồ Chí Minh và các tỉnh, thành phố lớn trên cả nước. Đặc điểm của công trình xây dựng nhà siêu cao tầng là có diện tích thi công chật hẹp, độ cao lớn, các yếu tố như gió và nhiệt độ tác động sẽ làm cho độ thẳng đứng của công trình không được đảm bảo. Để đảm bảo đúng yêu cầu kỹ thuật công tác trắc địa thi công xây dựng nhà siêu cao tầng đảm bảo độ thẳng đứng của công trình là hết sức cần thiết. Bài báo trình bầy một số giải pháp nâng cao hiệu quả công tác trắc địa thi công xây dựng nhà siêu cao tầng ở Việt Nam.

\section{1. Đặt vấn đề}

Ở nước ta khi thi công các công trình nhà cao tầng vẫn sử dụng máy kinh vĩ điện tử, toàn đạc điện tử, máy chiếu đứng. Trong đó, phương pháp sử dụng máy chiếu đứng cho độ chính xác cao hơn cả. Phương pháp này sử dụng tia ngắm thẳng đứng của máy chiếu quang học hoặc laze. Tia ngắm đó được tạo nên nhờ ống thủy chính xác hoặc bộ phận tự động cân bằng (cơ cấu điều hòa). Trong phương pháp này có hai cách chiếu: chiếu điểm liên tục với những công trình có chiều cao nhỏ và chiếu điểm theo bậc đối với những công trình có chiều cao lớn mỗi bậc chiếu là 10-20 tầng, trong quá trình chiếu điểm cần phải định vị vị trí các bậc chiếu.

Để đảm bảo yêu cầu kỹ thuật và thiết kế công trình thì các phương pháp bố trí thi công đảm bảo độ thẳng đứng của công trình là hết sức cần thiết. Vì vậy, cần phải nghiên cứu phương pháp nâng cao hiệu quả công tác trắc địa thi công xây dựng các công trình nhà siêu cao tầng ở Việt Nam.
2. Ứng dụng phương pháp chiếu theo bậc và định vị lại lưới trên các sàn thi cồng bằng công nghệ định vị vệ tinh

\subsection{Cơ sở lý thuyết của phương pháp}

Khi thi công xây dựng nhà cao tầng, để đảm bảo độ thẳng đứng của công trình cần phải thành lập trên mặt bằng móng công trình một lưới trắc địa chuyên dụng. Sau đó sử dụng máy chiếu đứng PZL (hoặc các máy chiếu có tính năng tương đương) đặt tại các điểm của lưới trắc địa chuyên dụng. Tại các sàn thi công trên cao dựa vào các điểm khống chế đã được chuyền lên, tiến hành đo kiểm tra các đại lượng góc và cạnh, tính toán bình sai và hoàn nguyên các điểm khống chế này về vị trí thiết kế để từ đó bố trí các hạng mục còn lại của công trình trên từng sàn thi công. Như vậy, độ chính xác thi công công trình theo phương thẳng đứng sẽ phụ thuộc vào chất lượng thành lập lưới trắc địa chuyên dụng trên mặt bằng móng công trình và chất lượng chiếu điểm khống chế lên các tầng cao. Hiện nay, có hai phương án chiếu điểm lên các sàn thi công

Ngày nhận bài: 05/02/2018, ngày chuyển phản biện: 08/02/2018, ngày chấp nhận phản biện: 28/02/2018, ngày chấp nhận đăng: 06/3/2018 
nhà siêu cao tầng.

- Phương án chiếu liên tục từ mặt sàn tầng 1 lên các tầng thi công: với phương án chiếu điểm này, chỉ cần đặt máy chiếu ở sàn tầng 1 chiếu điểm cho đển tầng cuối cùng. Khi số tầng của tòa nhà hay chiều cao công trình tăng lên thì độ chính xác chiếu điểm sẽ giảm đi do chiều cao tia ngắm dẫn đến sai số ngắm và sai số bắt mục tiêu và do ảnh hưởng của các yếu tố ngoại cảnh (gió, nhiệt độ,...) đến độ chính xác chiếu điểm.

Độ chính xác của lưới trắc địa chuyên dụng trên các sàn xây dựng tính theo công thức:

$$
m^{2}=m_{k c}^{2}+m_{c d}^{2}+m_{d d}^{2}
$$

trong đó: $m_{\mathrm{kc}}$ - sai số vị trí điểm lưới khống chế cơ sở trên mặt bằng móng;

$\mathrm{m}_{\mathrm{cd}}$ - sai số chiếu điểm;

$\mathrm{m}_{\mathrm{dd}}$ - sai số đánh dấu điểm

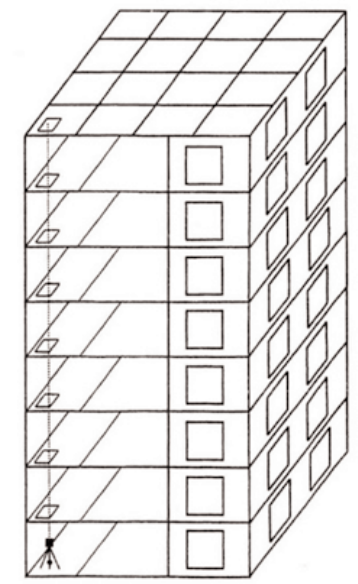

Hình 1: Sơ đồ chuyền tọa độ lên các sàn thi công bằng máy chiếu đứng

Trong công thức (1) ta có sai số ; $\mathrm{m}_{\mathrm{kc}}$ và $\mathrm{m}_{\mathrm{dd}}$ có giá trị ít thay đổi, vậy khi sai số $\mathrm{m}_{\mathrm{cd}}$ tăng thì sai số $m$ tăng, do đó, khi chiều cao tia ngắm tăng đến một giới hạn nào đó ta phải phân đoạn để chiếu nhằm đảm bảo sai số $\mathrm{m}$ nằm trong giới hạn cho phép.
- Phương án chiếu phân đoạn: chia số tầng thi công thành $n$ đoạn. Mỗi đoạn chiếu sẽ gồm $\mathrm{m}$ tầng. Tiến hành chiếu điểm từ tầng 1 đến hết đoạn thứ nhất bao gồm m tầng. Sau đó, chuyển máy chiếu lên tầng $\mathrm{m}$ và tiếp tục chiếu điểm cho đến hết tầng $2 \mathrm{~m}$. Chuyển máy chiếu lên tầng $2 \mathrm{~m}$ và tiến hành tương tự cho đến khi thi công xong công trình. Với phương án chiếu phân đoạn: sẽ giảm được chiều cao tia ngắm đi $\mathrm{n}$ lần, do đó, độ chính xác chiếu điểm trong từng đoạn sẽ tăng lên. Đây chính là phương pháp chuyền tọa độ lên các tầng thi công khi xây dựng các tòa nhà siêu cao tầng khi sử dụng các máy chiếu thiên đỉnh (PZL) hoặc các máy chiếu có tính năng tương đương để chuyền tọa độ lên các sàn thi công [4].

Tuy nhiên, trong phương pháp chiếu phân đoạn để đảm bảo độ chính xác thi công công trình theo phương thẳng đứng vấn đề đặc biệt quan trọng là cần xác định lại lại các điểm cơ sở của lưới trắc địa chuyên dụng tại tầng khởi đầu của mỗi đoạn chiếu.

Vấn đề định vị các điểm khống chế của lưới trắc địa chuyên dụng trên từng bậc chiếu chỉ thực hiện được nhờ công nghệ định vị vệ tinh.

\subsection{Quy trình chuyền tọa độ theo phương thẳng đứng theo phương pháp chiếu theo bậc}

Khi thi công xây dựng phần móng của công trình và thành lập lưới trắc địa chuyên dụng trên mặt bằng móng tại sàn tầng 1 , cần thành lập mạng lưới khống chế bằng công nghệ định vị vệ tinh (gọi là mạng lưới GNSS - được bố trí ở những vị trí ổn định trên mặt đất trong suốt quá trình thi công công trình). Các điểm trong mạng lưới GNSS được đo liên kết với nhau và liên kết với các điểm của lưới chuyên dụng. Các điểm trong lưới chuyên dụng liên kết với nhau bằng các trị đo góc, cạnh được xác định bằng các thiết bị đo đạc mặt đất (máy 
toàn đạc điện tử hoặc thước thép như trong hình 2).

Khi xử lý số liệu, tiến hành bình sai hỗn hợp các trị đo mặt đất (bao gồm các trị đo góc và cạnh trong lưới chuyên dụng được đo bằng máy toàn đạc điện tử hoặc thước thép) và các trị đo bằng công nghệ định vị vệ tinh theo thuật toán bình sai hỗn hợp lưới vệ tinh - mặt đất [1] ta có tọa độ thiết kế của các điểm trong lưới chuyên dụng (đặt tên là $\mathrm{M}, \mathrm{N}, \mathrm{P}, \mathrm{Q}$ ) trên các tầng thi công trong bậc chiếu đầu tiên. Khi kết thúc bậc mỗi bậc chiếu (chiếu đến tầng bội số của $\mathrm{m}$ ), ta tiến hành đo lặp lại mạng lưới GNSS và mạng lưới chuyên dụng (lúc này đã được chiếu lên sàn cuối của bậc chiếu - đặt tên là M', N', P', Q'). Tiến hành xử lý số liệu đo lặp, ta có tọa độ các điểm của lưới chuyên dụng $\left(X_{i}, Y_{i}\right)$ ở bậc chiếu thứ i. So sánh tọa độ của các điểm lưới chuyên dụng giữa bậc chiếu thứ i và bậc chiếu đầu tiên được độ lệch tọa độ theo công thức:

$$
\left.\begin{array}{rl}
\Delta X_{i} & =X_{i}-X_{1} \\
\Delta Y_{i} & =Y_{i}-Y_{1}
\end{array}\right\}
$$

Từ các đại lượng $\Delta X_{i}, \Delta Y_{i}$, ta tính được các đại lượng hoàn nguyên để hoàn nguyên các điểm ( $\left(\mathrm{M}^{\prime}, \mathrm{N}^{\prime}, \mathrm{P}^{\prime}, \mathrm{Q}^{\prime}\right)$ về đúng vị trí thiết kế làm cơ sở cho công tác bố trí chi tiết các hạng mục công trình trên các sàn thi công (hình 2).

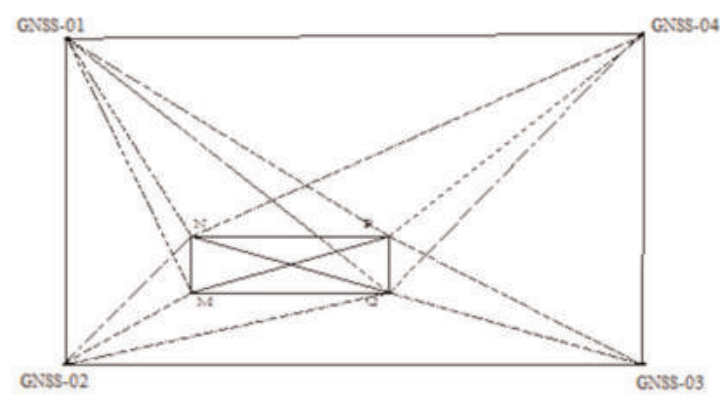

Hình 2: Sơ đồ lưới khống chế trắc địa

Rõ ràng là, khi kết hợp công nghệ định vị vệ tinh và máy chiếu đứng sẽ cho phép giảm chiều cao tia ngắm (bằng cách phân đoạn chiếu) làm tăng độ chính xác chuyền tọa độ lên các sàn thi công. Mặt khác, bằng kết quả đo lặp mạng lưới GNSS và lưới chuyên dụng trên các sàn thi công cho phép chúng ta kiểm tra và định vị lại các điểm lưới chuyên dụng tại tầng đầu tiên của các bậc chiếu trong quá trình thi công toàn bộ công trình. Đây chính là những đặc tính ưu việt của công nghệ định vị vệ tinh trong thi công xây dựng nhà siêu cao tầng ở nước ta.

\subsection{Vấn đề xác định trọng số của các trị đo trong bình sai hỗn hợp}

Với cùng số liệu đo, khi xử lý số liệu cần lưu ý đến phương pháp ước lượng trọng số các trị đo. Các phương pháp ước lượng trọng số khác nhau sẽ cho các kết quả xử lý số liệu khác nhau.

Trong bình sai hỗn hợp lưới mặt đất (gồm các trị đo mặt đất) và trị đo bằng công nghệ định vị vệ tinh (các baseline) theo phương pháp tính chuyển các baseline thành các gia số toạ độ phẳng thì ma trận trọng số đưa vào đối với các trị đo mặt đất là $\mathrm{P}_{\mathrm{t}}$, còn với các gia số toạ độ phẳng là $\mathbb{T}^{\mathrm{P}} \mathrm{u}$

Để ước lượng trọng số của các trị đo mặt đất, trước khi xử lý số, ta chỉ có thể dựa vào ước lượng sai số trung phương của các trị đo dựa theo một vài thông tin ban đầu như: loại máy đo, độ chính xác có trong lý lịch máy, số vòng đo, chất lượng máy đo.

Thông thường, sai số trung phương của các góc đo theo độ chính xác có trong lý lịch máy đo: $m_{\beta}, m_{S}$ [3]

Sau khi ước lượng được sai số trung phương của các trị đo chúng ta xác định trọng số theo công thức:

$$
P_{i}=\frac{C^{2}}{m_{i}^{2}}
$$

Trong đó, $\mathrm{m}_{\mathrm{i}}$ là sai số trung phương của trị đo thứ i. $\mathrm{C}$ là hằng số chọn tuỳ ý. 
Việc lựa chọn hằng số $\mathrm{C}$ trong tính trọng số các trị đo là tuỳ ý sao cho $\mathrm{P}$ trở thành con số thuận tiện và đơn giản khi xử lý số liệu đo. Tuy nhiên, hằng số $\mathrm{C}$ được chọn phải nhất quán với tất cả các trị đo.

Đối với các gia số toạ độ phẳng $A x^{\prime}, 4 y$ của các trị đo bằng công nghệ định vị vệ tinh, trọng số được tính từ ma trận tương quan $Q_{A z}$ tính chuyển từ ma trận hiệp phương sai của các trị đo $\Delta \mathrm{X}, \Delta \mathrm{Y}, \Delta \mathrm{Z}$ trong tệp tin giải cạnh.

$$
\mathrm{P}_{\Delta \mathrm{r}}=\mathrm{Q}^{-1} \Delta \mathrm{r}
$$

Ma trận tương quan QAx được lấy từ ma trận hiệp phương sai của các trị đo $\Delta \mathrm{X}, \Delta \mathrm{Y}, \Delta \mathrm{Z}$ trong công nghệ định vị vệ tinh[1].

Ta thấy rằng ma trận $\mathbb{T}^{3}$ : có các giá trị ngoài đường chéo khác không. Điều này chứng tỏ các giá trị gia số toạ độ phẳng

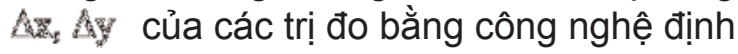
vị vệ tinh là các trị đo phụ thuộc.

Việc đưa các giá trị gia số toạ độ phẳng

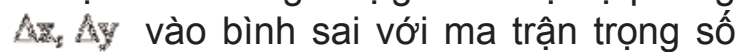
$\mathbb{P}^{3}$ :à có thể lấy là ma trận đầy đủ các thành phẩn ngoài đường chéo hoặc chỉ các thành phần trên đường chéo chính. Kết quả nghiên cứu, khảo sát và tính toán thực nghiệm đã chứng minh rằng: hiện nay các trị đo bằng công nghệ định vị về tinh được đo với độ chính xác cao hơn nhiều lần trị đo mặt đất nên ảnh hưởng của sự phụ thuộc các gia số toạ độ phẳng $\Delta \mathrm{Ax}_{\mathrm{z}} \mathrm{Ay}$ tính từ trị đo bằng công nghệ định vị vệ tinh đến kết quả xử lý số liệu hỗn hợp là không đáng kể, có thể bỏ qua [2]. Điều này có nghĩa là trọng số của các gia số toạ độ phẳng $A \mathrm{Ax}^{\mathrm{s}} \mathrm{Ay}$ của các trị đo bằng công nghệ định vị vệ tinh khi đưa vào xử lý số liệu hổn hợp chỉ cần lấy các thành phần trên đường chéo chính của ma trận $\mathbb{T}_{\mathcal{H}}^{\mathrm{H}}$.

Qua kết quả đo đạc xử lý số liệu thực nghiệm, khi xử lý số liệu hỗn hợp lưới vệ tinh và mặt đất thuần túy, sai số trung phương đơn vị trọng số $\mu$ thường khác xa 1 , điều này cho thấy trọng số của các trị đo bằng công nghệ định vị vệ tinh khi đưa vào xử lý số liệu phản ánh không đúng sai số đo.

Để xử lý trọng số trong bình sai hỗn hợp, cần thực hiện bình sai theo hai bước:

- Bước 1: Xử lý số liệu hỗn hợp trị đo vệ tinh - mặt đất thuần túy để xác định tọa độ sơ bộ và xác định sai số trung phương đơn vị trọng số $\mu_{1}$ của bình sai bước 1 .

- Bước 2: Xử lý số liệu hỗn hợp trị đo vệ tinh - mặt đất, trong đó chuẩn hóa lại trọng số của các trị đo bằng công nghệ định vị vệ tinh bằng cách nhân thêm $\left(\frac{1}{\mu_{1}^{2}}\right)$.

trong đó: $\mu_{1}$ là sai số trung phương trọng số đơn vị được lấy từ kết quả bình sai lần thứ nhất [5].

Sau khi xử lý số liệu lưới trắc địa chuyên dụng trên các sàn thi công sẽ xác định được chuyển dịch của các điểm khống chế trong lưới so với vị trí thiết kế trên mặt bằng móng công trình. Tiến hành hoàn nguyên các điểm khống chế về vị trí thiết kế và sử dụng các điểm này làm cơ sở cho các bậc chiếu tiếp theo.

\section{Kết quả thực nghiệm}

Để kiểm chứng các kết quả nghiên cứu lý thuyết đã nêu, chúng tôi đã tiến hành thực nghiệm đo đạc và xử lý số liệu mạng lưới chuyển trục công trình lên sàn thi công đo bằng công nghệ định vị vệ tịh và máy toàn đạc điện tử.

\subsection{Nội dung thực nghiệm}

Ngày 02 tháng 05 năm 2016, công tác thực nghiệm lưới trắc địa chuyên dụng bằng công nghệ định vị vệ tịnh và toàn đạc điện tử đã được tiến hành tại Viện KHCN Xây dựng, số 81 Trần Cung, Hà Nội. 
Mạng lưới đo đạc thực nghiệm bao gồm 04 điểm của lưới chiếu chuyên dụng $(\mathrm{M}, \mathrm{N}$, $\mathrm{P}, \mathrm{Q})$ được bố trí trên tầng mái của công trình và 03 điểm lưới GNSS dưới mặt đất (GNSS-01, GNSS-02, GNSS-03) được bố trí tại những vị trí đảm bảo việc thu tín hiệu vệ tinh.

Để kiểm tra độ chính xác thực tế khi đo đạc mạng lưới bằng công nghệ định vị vệ tinh kết hợp trị đo mặt đất, tại các vị trí điểm $\mathrm{M}, \mathrm{N}, \mathrm{P}, \mathrm{Q}$ chúng tôi thiết lập thêm 4 điểm $\mathrm{M} 1, \mathrm{~N} 1, \mathrm{P} 1, \mathrm{Q} 1$. Trong đó, các điểm $\mathrm{M} 1, \mathrm{~N} 1$, $\mathrm{P} 1, \mathrm{Q} 1$ cách các điểm $\mathrm{M}, \mathrm{N}, \mathrm{P}, \mathrm{Q}$ một khoảng từ 4 đến $5 \mathrm{~mm}$. Các giá trị dịch chuyển này được xác định chính xác bằng thước thép. Với giả thiết các điểm $\mathrm{M}, \mathrm{N}, \mathrm{P}$, $Q$ là các điểm lưới chiếu chuyên dụng tại mặt bằng tầng 1 , các điểm $\mathrm{M} 1, \mathrm{~N} 1, \mathrm{P} 1, \mathrm{Q} 1$ là các điểm được chiếu lên từ mặt bằng tầng 1 bằng máy chiếu đứng $\mathrm{PZL}$ (các điểm này có sai lệch so với tầng 1 do sử dụng phương pháp chiếu đứng gây ra lần lượt là $5 \mathrm{~mm}, 5 \mathrm{~mm}, 5 \mathrm{~mm}, 5 \mathrm{~mm})$. Sử dụng 07 máy thu tín hiệu vệ tinh Trimble R3 đo đạc 2 ca, thời gian đo mỗi ca 90 phút.

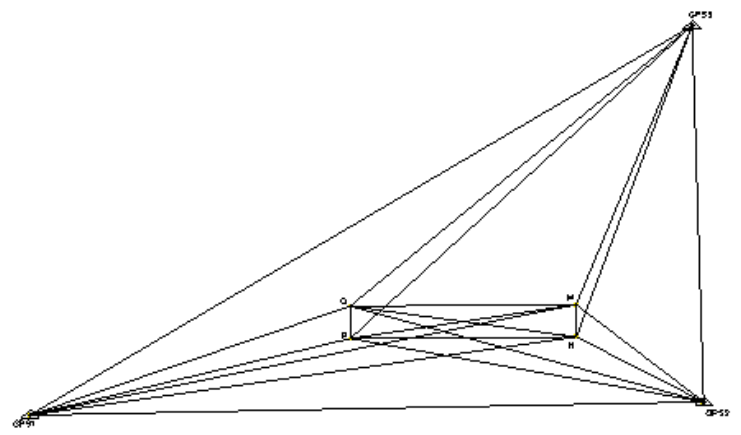

Hình 3: So đồ mạng lưới GNSS - mặt đất đo đạc thực nghiệm

Sử dụng thước thép để đo bổ sung chiều dài cạnh giữa các điểm lưới chiếu chuyên dụng trên mặt sàn thi công.

Như vậy, sẽ tạo thành mạng lưới hỗn hợp độc lập nhau: gồm 07 điểm GPS1, GPS2, GPS3, M1, N1, P1, Q1: có 18 vector cạnh (Baseline): GPS1-M1, GPS1-N1, GPS1-P1, GPS1-Q1, GPS2-M, GPS2-N,
GPS2-P, GPS2-Q1, GPS3-M1, GPS3-N1, GPS3-P1, GPS3-Q1, M1-N1, N1-P1, P1Q1, Q1-M1, M1-P1, N1-Q1 và 06 cạnh toàn đạc: M1-N1, N1-P1, P1-Q1, Q1-M1, M1-P1, N1-Q1.

\subsection{Kết quả đo thụ̂c nghiệm}

Sau khi đo đạc xong tại thực địa, tiến hành xử lý cạnh và bình sai mạng lưới GNSS bằng phần mềm TBC 2.0 (Trimble Business Center 2.0) cho từng mạng lưới, hệ tọa độ sử dụng để xử lý số liệu là hệ tọa độ VN2000, kinh tuyến trục 10500' múi chiếu $3^{\circ}$. Từ kết quả giải cạnh của các vector cạnh GNSS kết hợp với các trị đo chiều dài cạnh tiến hành xử lý số liệu hỗn hợp cho từng mạng lưới bằng phần mềm BSHH v1.0

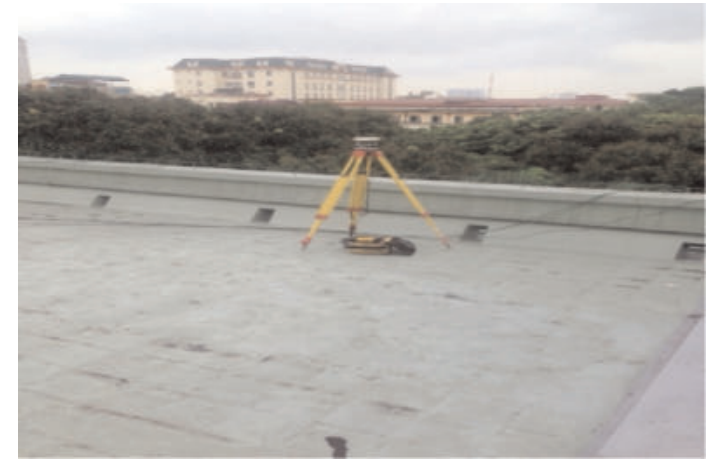

Hình 4: Thu tín hiệu vệ tinh bằng máy Trimble R3

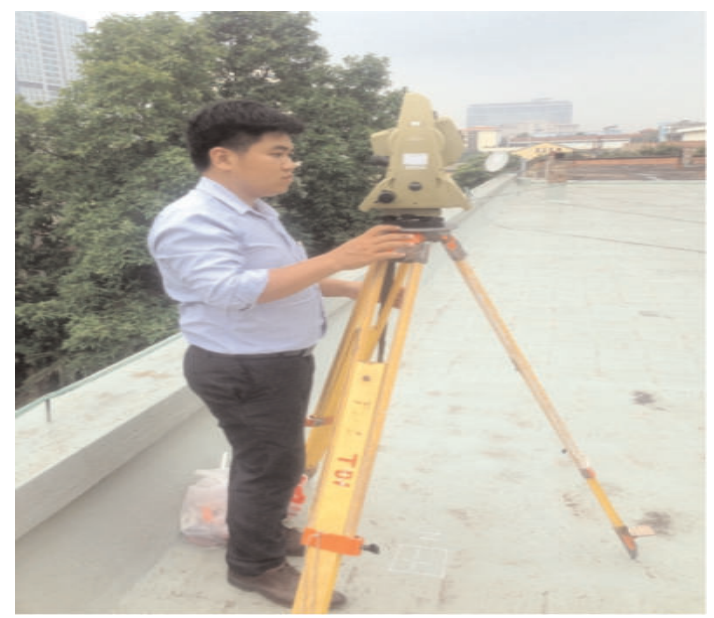

Hình 5: Đo cạnh bằng máy TC-1800 
4. Kết quả xử lý lưới hỗn hợp vệ tinh và các cạnh lưới mặt đất đo bằng thước thép

Sử dụng thước thép để đo bổ sung chiều dài cạnh giữa các điểm lưới chiếu chuyên dụng trên mặt sàn thi công ta được kết quả đo cạnh như cột 2 (bảng 1 và bảng 2). Kết quả đo cạnh lưới bằng công nghệ định vị vệ tinh sau khi đã xử lý ghi trong cột 3 .

Bảng 1: So sánh chiều dài cạnh mạng lưới 1

\begin{tabular}{|c|c|c|c|}
\hline $\begin{array}{c}\text { Tên } \\
\text { cạnh }\end{array}$ & $\begin{array}{c}\text { Cạnh đo bằng } \\
\text { thước thép } \\
(\mathrm{m})\end{array}$ & $\begin{array}{c}\text { Cạnh đo bằng } \\
\text { GPS }(\mathrm{m})\end{array}$ & $\Delta \mathrm{S}$ \\
\hline $\mathrm{M}-\mathrm{N}$ & 6.201 & 6.202 & 0.001 \\
\hline $\mathrm{M}-\mathrm{P}$ & 36.817 & 36.821 & 0.004 \\
\hline $\mathrm{M}-\mathrm{Q}$ & 36.292 & 36.295 & 0.003 \\
\hline $\mathrm{N}-\mathrm{P}$ & 36.290 & 36.292 & 0.002 \\
\hline $\mathrm{N}-\mathrm{Q}$ & 36.815 & 36.817 & 0.002 \\
\hline $\mathrm{P}-\mathrm{Q}$ & 6.199 & 6.197 & -0.002 \\
\hline
\end{tabular}

Bảng 3: Tọa độ sau bình sai và sai số vị trí điểm của mạng lưới 1 xử lý bằng phần mềm BSHH v1.0
Bảng 2: So sánh chiều dài cạnh mạng lưới 2

\begin{tabular}{|c|c|c|c|}
\hline $\begin{array}{c}\text { Tên } \\
\text { cạnh }\end{array}$ & $\begin{array}{c}\text { Cạnh đo bằng } \\
\text { thước thép } \\
(\mathrm{m})\end{array}$ & $\begin{array}{c}\text { Cạnh đo bằng } \\
\text { GPS }(\mathrm{m})\end{array}$ & $\Delta \mathrm{S}$ \\
\hline $\mathrm{M} 1-\mathrm{N} 1$ & 6.203 & 6.202 & -0.001 \\
\hline $\mathrm{M} 1-\mathrm{P} 1$ & 36.817 & 36.820 & 0.003 \\
\hline $\mathrm{M} 1-\mathrm{Q} 1$ & 36.292 & 36.296 & 0.004 \\
\hline $\mathrm{N} 1-\mathrm{P} 1$ & 36.289 & 36.291 & 0.002 \\
\hline $\mathrm{N} 1-\mathrm{Q} 1$ & 36.816 & 36.819 & 0.003 \\
\hline $\mathrm{P} 1-\mathrm{Q} 1$ & 6.198 & 6.199 & 0.001 \\
\hline
\end{tabular}

* Đánh giá độ chính xác mạng lưới đo đạc thực nghiệm

Từ giá trị sai số thực (cột thứ 4 trong bảng 5) có thể tính được sai số trung phương chuyền tọa độ lên sàn thi công:

$$
m=\sqrt{\frac{[\Delta \Delta]}{n}}
$$

\begin{tabular}{|c|c|c|c|c|c|c|c|}
\hline STT & $\begin{array}{c}\text { Tiêu } \\
\text { điểm }\end{array}$ & $\mathrm{X}_{\mathrm{bs}}(\mathrm{m})$ & $\mathrm{Y}_{\mathrm{bs}}(\mathrm{m})$ & $\mathrm{m}_{\mathrm{X}}$ & $\mathrm{M}_{Y}$ & $\mathrm{M}_{\mathrm{P}}$ & Ghi chú \\
\hline 1 & $\mathrm{M}$ & 2328560.0777 & 581857.2805 & 0.001 & 0.002 & 0.003 & \\
\hline 2 & $\mathrm{~N}$ & 2328553.8767 & 581857.3307 & 0.001 & 0.002 & 0.003 & \\
\hline 3 & $\mathrm{P}$ & 2328553.5706 & 581821.0425 & 0.001 & 0.002 & 0.003 & \\
\hline 4 & $\mathrm{Q}$ & 2328559.7683 & 581820.9898 & 0.001 & 0.002 & 0.003 & \\
\hline
\end{tabular}

Bảng 4: Tọa độ sau bình sai và sai số vị trí điểm của mạng lưới 2 bình sai bằng phần mềm $B S H H$ v1.0

\begin{tabular}{|c|c|c|c|c|c|c|c|}
\hline STT & $\begin{array}{c}\text { Tiêu } \\
\text { điểm }\end{array}$ & $\mathrm{X}_{\mathrm{bs}}(\mathrm{m})$ & $\mathrm{Y}_{\mathrm{bs}}(\mathrm{m})$ & $\mathrm{m}_{\mathrm{X}}$ & $\mathrm{M}_{\mathrm{Y}}$ & $\mathrm{M}_{\mathrm{P}}$ & Ghi chú \\
\hline 1 & $\mathrm{M} 1$ & 2328560.083 & 581857.274 & 0.0008 & 0.0013 & 0.0015 & \\
\hline 2 & $\mathrm{~N} 1$ & 2328553.881 & 581857.325 & 0.0008 & 0.0013 & 0.0015 & \\
\hline 3 & $\mathrm{P} 1$ & 2328553.574 & 581821.036 & 0.0008 & 0.0013 & 0.0016 & \\
\hline 4 & $\mathrm{Q} 1$ & 2328559.771 & 581820.983 & 0.0008 & 0.0013 & 0.0016 & \\
\hline
\end{tabular}


Bảng 5: Tổng hợp kết quả thực nghiệm bình sai bằng phần mềm BSHH v1.0

\begin{tabular}{|c|c|c|c|}
\hline \multirow{2}{*}{ Tiêu điểm } & Độ lệch thực & $\begin{array}{c}\text { Độ lệch xác định bằng tọa độ sau bình sai } \\
\text { của BSHH v1.0 }\end{array}$ & $\begin{array}{c}\text { Sai lệch } \\
\text { (sai số thực) } \\
\text { (mm) }\end{array}$ \\
\cline { 2 - 4 } & Khoảng cách $(\mathrm{mm})$ & Khoảng cách $(\mathrm{mm})$ & 3.4 \\
\cline { 3 - 4 } & 5.0 & 8.4 & 2.1 \\
\hline M-M1 & 5.0 & 7.1 & 2.3 \\
\hline N-N1 & 5.0 & 7.3 & 2.3 \\
\hline P-P1 & 5.0 & 7.3 & \\
\hline Q-Q1 & & & \\
\hline
\end{tabular}

trong đó: $\Delta$ là sai số thực $\mathrm{n}$ là số điểm đo

Từ kết quả bình sai bằng phần mềm BSHH v1.0, tính được sai số trung phương chuyển tọa độ lên sàn thi công bằng công nghệ định vị vệ tinh kết hợp trị đo mặt đất là:

$$
m= \pm \sqrt{\frac{\left.3.4^{2}+2.1^{2}+2.3^{2}+2.3^{2}\right)}{4}}= \pm 2.58 \mathrm{~mm}
$$

So sánh với hạn sai cho phép sai số trung phương chuyển trục và độ cao lên các mặt bằng xây lắp, ta thấy sai số thực nhỏ hơn hạn sai cho phép.

Từ kết quả đo đạc và tính toán thực nghiệm ta thấy:

- Công nghệ định vị vệ tinh có đủ độ chính xác để đo kiểm tra và định vị vị trí các điểm của lưới trắc địa chuyên dụng đã được chiếu lên các sàn thi công trên các tầng cao.

- Các giá trị độ lệch xác định được từ kết quả xử lý số liệu hỗn hợp lưới vệ tinh và mặt đất phù hợp với giá trị độ lệch thực tế.

- Từ kết quả đo đạc và xử lý số liệu lưới thực nghiệm cho thấy khi chuyển điểm lên sàn thi công bằng công nghệ định vị vệ tinh kết hợp với các trị đo mặt đất có độ chính xác hoàn toàn đảm bảo các yêu cầu kỹ thuật cần thiết để xác định các giá trị độ lệch của các điểm lưới chiếu chuyên dụng được chiếu lên sàn đầu tiên của mỗi bậc chiếu bằng máy chiếu đứng. Phương pháp này cho phép nâng cao hiệu quả của công tác trắc địa trong bố trí thi công xây dựng nhà siêu cao tầng ở nước ta.

\section{Kết luận và kiến nghị}

\subsection{Kết luận}

Từ những kết quả nghiên cứu lý thuyết và thực nghiệm một số kết luận được rút ra như sau:

1. Để nâng cao độ chính xác thi công các công trình nhà siêu cao tầng, nên sử dụng phương án chiếu theo bậc và ứng dụng công nghệ định vị vệ tinh để định vị lại vị trí của các điểm của lưới chuyên dụng tại các bậc chiếu.

2. Khi sử dụng mạng lưới hỗn hợp vệ tinh- mặt đất có thể phát hiện chuyển dịch của các điểm khống chế của lưới trắc địa chuyên dụng. Vì vậy, để nâng độ chính xác thi công các công trình nhà siêu cao tầng cần kết hợp giữa công nghệ định vị vệ tinh, trị đo mặt đất và máy chiếu đứng. Bằng kết quả định vị vệ tinh kết hợp với các trị đo mặt đất cho phép chúng ta kiểm tra và định vị lại các điểm lưới chiếu chuyên dụng tại tầng đầu tiên của các bậc chiếu trong quá trình thi công toàn bộ công trình. Điều này sẽ góp phần nâng cao độ chính xác thi công các 
công trình có chiều cao lớn.

\subsection{Kiến nghị}

Nội dung công tác trắc địa trong đảm bảo thi công xây dựng công trình nhà siêu cao tầng rất đa dạng và phức tạp. Vì vậy, cần phải tiếp tục nghiên cứu phát triển và hoàn thiện việc ứng dụng các công nghệ đo đạc tiên tiến nhằm nâng cao độ chính xác thi công xây dựng các công trình nhà siêu cao tầng ở nước ta. $\bigcirc$

\section{Tài liệu tham khảo}

[1]. Hoàng Ngọc Hà (2006), Bình sai tính toán lưới trắc địa và GPS, NXB Khoa học và kỹ thuật, Hà Nội.

[2]. Hoàng Ngọc Hà (2006), "Một số vấn đề về các mô hình toán học bình sai hỗn hợp lưới mặt đất - GPS", Tuyển tập công trình khoa học - chuyên đề kỷ niệm 40 năm thành lập khoa trắc địa, Trường Đại học Mỏ địa chất, Hà Nội.

[3]. Diêm Công Huy (2014), "Nghiên cứu kết hợp công nghệ GPS và thiết bi chiếu đứng để đảm bảo độ thẳng đứng trong xây dựng nhà siêu cao tầng ở Việt Nam", Luận văn thạc sỹ kỹ thuật, Đại học mỏ - địa chất, Hà Nội.

[4]. Nguyễn Quang Thắng, Trần Viết Tuấn (2009), Trắc địa công trình công nghiệp - thành phố, Bài giảng ngành Trắc địa, Đại học Mỏ-Địa chất, Hà Nội.

[5]. Lại Nam Thái (2014), "Nghiên cứu một số giải pháp kỹ thuật nhằm nâng cao hiệu quả công tác xây dựng các công trình có chiều cao lớn Việt Nam", Luận văn thạc sỹ kỹ thuật, Đại học mỏ - địa chất, Hà Nội. $\bigcirc$

\section{Summary}

Study some methods to improve the efficiency of construction surveying for the skyscrapers in Vietnam

Diem Cong Trang, Lai Nam Thai

Vietnam Institute for Building Science and Technology

Pham Thanh Thao

Hanoi University of Mining and Geology

In recent years, the skyscrapers construction projects are widely deployed in Hanoi, Ho Chi Minh and other big cities nationwide of Vietnam. The characteristics of the skyscraper Site building construction includes narrow construction, high altitude, and such impact as wind and temperature will make the verticality of the building work un-guaranteed. It is very necessary to conduct construction surveying of skyscrapers project to ensure the verticality of the work and the correct implemention according to the technical requirements and construction design. The peper present some methods to improve the efficiency of construction surveying for the skyscrapers in Vietnam. $O$ 